\title{
A note on Candido's identity and Heron's formula
}

\author{
Darko Veljan \\ Department of Mathematics, University of Zagreb \\ Bijenička c. 30, 10002 Zagreb, CROATIA \\ e-mail : darko.veljan@gmail.com
}

\begin{abstract}
The square of the sum of squares of three consecutive Fibonacci numbers is twice the sum of their fourth powers. This is Candido's identity. Abstractly, it holds for any two elements and their sum in any commutative ring. Geometrically, it reveals the degeneracy of a triangle via Heron's area formula. Higher dimensional analogues via volume formulas of tetrahedra are also discussed. The identity and its cubic variant can be applied to various combinatorial and geometric formulas to obtain corresponding symmetric identities, and an algebraic application is to the polynomial Fermat's cubic equation.
\end{abstract}

Keywords: Candido's identity; Heron's formula; Fibonacci numbers

MSC: 11B39

\subsection{Candido's identity and some applications}

The Candido identity (named after Giacomo Candido, 1871-1941) was published in 1951, and says that the square of the sum of squares of three consecutive Fibonacci numbers is equal twice the sum of their fourth powers. Thus,

$$
\left(F_{n-1}^{2}+F_{n}^{2}+F_{n+1}^{2}\right)^{2}=2\left(F_{n-1}^{4}+F_{n}^{4}+F_{n+1}^{4}\right) .
$$

DOI: https://doi.org/10.5592/CO/CCD.2016.08 
To prove it geometrically, recall first that the area $S$ of a triangle $\triangle A B C$ with side lengths $a, b$ and $c$ is given by Heron's formula from about $60 \mathrm{AD}$, but apparently Archimedes knew it some 300 years earlier. It reads as follows.

$$
\begin{aligned}
(4 S)^{2} & =\left(a^{2}+b^{2}+c^{2}\right)^{2}-2\left(a^{4}+b^{4}+c^{4}\right)=(2 a b)^{2}-\left(a^{2}+b 2-c^{2}\right)^{2} \\
& =(a+b+c)(-a+b+c)(a-b+c)(a+b-c) .
\end{aligned}
$$

(A quick proof of Heron's formula is by using Pythagoras' theorem $\cos ^{2} C+\sin ^{2} C=1$, and cosine and sine rules: $\cos C=\left(a^{2}+b^{2}-c^{2}\right) /(2 a b)$ and $\sin C=2 S /(a b)$.) Now, from the basic Fibonacci recurrence $F_{n+1}=F_{n}+F_{n-1}$, it follows that the triangle whose side lengths are these numbers is degenerated. Hence, its area $S$ is zero. By Heron's formula, the difference between the left and right side of Candido's identity is zero. This proves the identity.

By the same geometric argument, Candido's identity holds for any two positive numbers and their sum. For a genuine triangle, of course, we have the corresponding Candido's inequality.

In a pure algebraic setting, for every two elements $x, y$ of any commutative ring, the Candido identity is a curious equality (and can simply be checked by expanding both sides):

$$
\left(x^{2}+y^{2}+(x+y)^{2}\right)^{2}=2\left(x^{4}+y^{4}+(x+y)^{4}\right) .
$$

For three summands, $x+(y+z)=(x+y)+z$, we obtain two corresponding Candido identities etc.

Interpretations and visual proofs of the identity for real positive numbers are given in [1] and [2]. We remark that Candido's identity and the basic Fibonacci recurrence are equivalent, again by Heron's formula. Note that this identity also holds for Lucas numbers, Pell numbers, hyper-Fibonacci numbers (defined for $n, r \geq 1$ by $F_{n}^{(r)}=F_{n-1}^{(r)}+$ $F_{n}^{(r-1)}$, and $F_{n}^{(0)}=F_{n}, F_{0}^{(r)}=0, F_{1}^{(r)}=1$, for $\left.n, r \geq 0\right)$, Horadam polynomial second order recurrences, and many other sequences of that type. In fact, it holds for every sequence defined by recurrence or formula of the form $x+y=z$.

A generalization of Candido's identity is the following functional equation for an unknown function $f$ defined on positive real numbers with values also in positive reals:

$$
f[f(x)+f(y)+f(x+y)]=2[f(f(x))+f(f(y))+f(f(x+y))] .
$$

The only solution when $f$ is a continuous surjection with $f(0)=0$ is the squaring function (times a constant), i.e., $f(x)=C x^{2}$, see [1]. Proof boils down to prove that the 
set $\left\{2^{m} 3^{n}: m, n\right.$ integers $\}$ is dense on positive reals, and this follows from the fact that the sequence $\log _{2} 3,2 \log _{2} 3,3 \log _{2} 3, \ldots$ of multiples of the transcendental number $\log _{2} 3$ is uniformly distributed modulo 1.

Candido's identity simply transforms a recurrence or formula (automatically) in terms of sums of squares and fourth powers or in terms of other symmetric functions. For example, consider combinatorially interesting Padovan's sequence $1,1,1,2,2,3,4,5,7,9,12,16,21, \ldots$ where each member (except first three) is the sum of the second and third preceding it; $P_{n+2}=P_{n}+P_{n-1}, P_{0}=P_{1}=P_{2}=1$. The $n$-th Padovan number $P_{n}, n \geq 2$, is the number of ways of writing $n+2$ as an ordered sum (composition) of 2's and 3's. (Recall, $F_{n+1}, n \geq 1$, is the number of compositions of 1 's and 2's whose sum is $n$.) In the same manner as before, we have

$$
\left(P_{n-1}^{2}+P_{n}^{2}+P_{n+2}^{2}\right)^{2}=2\left(P_{n-1}^{4}+P_{n}^{4}+P_{n+2}^{4}\right) .
$$

As the golden ratio is attached to Fibonacci numbers, consecutive Padovan numbers ratios tend to the plastic number $p$ (approximately 1,3247...), satisfying the cubic equation $p^{3}=p+1$. Yet another combinatorial example is to apply Candido's identity to Pascal's formula for binomial coefficients $\left(\begin{array}{c}n+1 \\ k\end{array}\right)=\left(\begin{array}{c}n \\ k-1\end{array}\right)+\left(\begin{array}{l}n \\ k\end{array}\right)$ to get a symmetric expression in all three of them. So, we get "for free" the following identity:

$$
\left[\left(\begin{array}{c}
n \\
k-1
\end{array}\right)^{2}+\left(\begin{array}{l}
n \\
k
\end{array}\right)^{2}+\left(\begin{array}{c}
n+1 \\
k
\end{array}\right)^{2}\right]^{2}=2\left[\left(\begin{array}{c}
n \\
k-1
\end{array}\right)^{4}+\left(\begin{array}{c}
n \\
k
\end{array}\right)^{4}+\left(\begin{array}{c}
n+1 \\
k
\end{array}\right)^{4}\right] .
$$

A geometric example is to apply Candido's identity to the Pythagorean theorem $\left(c^{2}=\right.$ $\left.a^{2}+b^{2}\right)$. The result is totally symmetric. So, a triangle is right if and only if the square of the sum of fourth powers of its side lengths is twice the sum of their eight powers:

$$
\left(a^{4}+b^{4}+c^{4}\right)^{2}=2\left(a^{8}+b^{8}+c^{8}\right) .
$$

Denoting by $p_{n}$ the sum of $n$-th powers in variables $a, b$ and $c$, the Pythagorean theorem then reads as follows:

$$
p_{4}^{2}=2 p_{8} .
$$

The form $a^{2}+b^{2}=c^{2}$ needs 8 symbols, while the above version needs only 7 symbols $(5$ different). Note that degenerate Heronian triangles (integer side lengths and area zero) are not accounted by parametrizations given by Brahmagupta and later by Euler (see $[3]$ ). Of course, they are parametrized by two nonnegative integers (and their sum). 


\subsection{The cubic Candido's identity, Fermat's equality and other appli- cations}

Heron's formula can also be written in the following way. Let $e_{1}=a+b+c=2 s$ (perimeter), $e_{2}=a b+b c+c a, e_{3}=a b c$, be the elementary symmetric functions of $a, b$ and $c$. Then from

$$
(4 S)^{2}=2 s(2 s-2 a)(2 s-2 b)(2 s-2 c)=e_{1}\left(4 e_{1} e_{2}-e_{1}^{3}-8 e_{3}\right)
$$

we get that $S=0$ if and only if $4 e_{1} e_{2}-e_{1}^{3}-8 e_{3}=0$ (assuming non degeneracy $e_{1} \neq 0$ ). So, $D_{3}(a, b, c):=(-a+b-c)(a-b+c)(a+b-c)=0$ implies the cubic Candido's identity

$$
(a+b+c)^{3}+8 a b c=4(a+b+c)(a b+b c+c a) .
$$

This identity also makes sense in any integral domain (commutative ring without zero divisors), for elements $a, b, c$ whose sum is not zero, but one of them is the sum of the other two. In geometric terms of the semi-perimeter $s$ of a triangle, it can simply be written as:

$$
s^{3}+e_{3}=s e_{2} .
$$

Hence, we have the corresponding cubic Candido identities for Fibonacci numbers, Padovan numbers, Pascal's formula, Horadam polynomials, etc. before substitution $a+b=c$.

For any triangle (genuine or degenerate) we have the cubic Candido's triangle inequality:

$$
s^{3}+a b c \leq s(a b+b c+c a)
$$

with equality if and only if the triangle is degenerate (just multiply all three triangle inequalities). Algebraically, Candido's identity $p_{2}^{2}=2 p_{4}$ is equivalent to $e_{1}\left(e_{1}^{3}-4 e_{1} e_{2}+\right.$ $\left.8 e_{3}\right)=0$, and this follows simply by expressing power sums $p_{2}$ and $p_{4}$ in terms of $e_{1}, e_{2}$ and $e_{3}$.

As a combinatorial example this time, consider the sequence $1,1,2,4,9,21,51,127,323, \ldots$ of Motzkin numbers $M_{n}$ given by

$$
(n+2) M_{n}=(2 n+1) M_{n-1}+3(n-1) M_{n-2},
$$

and $M_{0}=M_{1}=1$. The Motzkin number $M_{n+1}$ counts $n$-tuples of positive integers which start and end by either 1 or 2 with differences between neighbors $-1,0$, or 1 . The 
quotients $M_{n+1} / M_{n} \rightarrow 3$ as $n \rightarrow \infty$. By the cubic Candido's identity we have (again "for free") the corresponding identity for Motzkin numbers:

$$
\begin{array}{r}
\left((n+2) M_{n}+(2 n+1) M_{n-1}+3(n-1) M_{n-2}\right)^{3}+24(n+2)(2 n+1)(n-1) M_{n} M_{n-1} M_{n-2} \\
=4\left[(n+2) M_{n}+(2 n+1) M_{n-1}+3(n-1) M_{n-2}\right] \\
\times\left[\left(2 n^{2}+5 n+2\right) M_{n} M_{n-1}+3\left(n^{2}+n-2\right) M_{n} M_{n-2}+3\left(2 n^{2}-n-1\right) M_{n-1} M_{n-2}\right] .
\end{array}
$$

If we apply Candido's cubic identity to the Pythagorean theorem $a^{2}+b^{2}=c^{2}$, now with $e_{1}=a^{2}+b^{2}+c^{2}, e_{2}=a^{2} b^{2}+b^{2} c^{2}+c^{2} a^{2}, e_{3}=a^{2} b^{2} c^{2}$, then $s^{3}+e_{3}=s e_{2}$ implies the following symmetric identity of degree 6 (before substituting $a^{2}+b^{2}=c^{2}$ ):

$$
\left(a^{2}+b^{2}+c^{2}\right)^{3}+8(a b c)^{2}=4\left(a^{2}+b^{2}+c^{2}\right)\left((a b)^{2}+(b c)^{2}+(c a)^{2}\right) .
$$

We can apply Candido's cubic identity to the Ptolemy theorem $(e f=a c+b d$ for a circumscribed quadrilateral with side lengths $a, b, c, d$ and diagonals $e, f)$, and many other basic geometric facts equivalent to the Euclidean fifth postulate, but we shall skip it here.

Consider now the cubic ("Fermat") equality $a^{3}+b^{3}=c^{3}$ for relatively prime polynomials $a=a(x)$ etc. over integers, and at least one of the polynomials $a, b, c$ non constant. From cubic Candido's identity $e_{1}^{3}+8 e_{3}=4 e_{1} e_{2}$, we have

$$
\left(a^{3}+b^{3}+c^{3}\right)^{3}+(2 a b c)^{3}=4\left(a^{3}+b^{3}+c^{3}\right)\left((a b)^{3}+(b c)^{3}+(c a)^{3}\right) .
$$

In the ring of integer polynomials we have two relatively prime polynomial factorizations into symmetric homogeneous irreducible factors of degrees 3 and 6 in variables $a, b, c$ (before substituting $a^{3}+b^{3}=c^{3}$ ):

$$
\begin{array}{r}
\left(a^{3}+b^{3}+c^{3}+2 a b c\right)\left(\left(a^{3}+b^{3}+c^{3}\right)^{2}-2(a b c)\left(a^{3}+b^{3}+c^{3}\right)+4(a b c)^{2}\right) \\
=4\left(a^{3}+b^{3}+c^{3}\right)\left((a b)^{3}+(b c)^{3}+(c a)^{3}\right) .
\end{array}
$$

If we write it as $P Q=4 R S$, the only possibilities are $P=k R, Q=l S$, where $k, l$ are integers with $k l=4$. For $x=0$ denote $a_{0}=a(0)$ etc. Then $a_{0}^{3}+b_{0}^{3}=c_{0}^{3}$, where we may assume that $a_{0}, b_{0}, c_{0}$ are coprime integers. So, only one of them is even. Say, $a_{0}$ is even and $b_{0}, c_{0}$ odd. If $k=l=2$, i.e., $P=2 R, Q=2 S$, we get $2(a b c)^{2}=(a b)^{3}+\left(a_{3}+b_{3}\right)^{2}$, and for $x=0$, on the left is an even number, and on the right an odd number, a contradiction. If $k=1$ (and $l=4$ ), then $a b c=0$, say $a=0$, and this contradicts the fact that $a, b, c$ are coprime. If $k=4$ (and $l=1$ ), and $k=-1, k=-2$ and $k=-4$, the even-odd game brings us again to absurds. The same argument works not only for cubes but also for all multiple powers of 3 . 
Let us only briefly recall that in general, if $f, g$ and $h$ are relatively prime polynomials (over complex numbers), at least one of them non constant, and such that $f^{n}+g^{n}=h^{n}$, then $n \leq 2$. To prove it, recall first Mason's lemma (from 1984): if $a+b=c$, where $a=a(x), b=b(x), c=c(x)$ are coprime polynomials, at least one of them non constant, then $\max \{\operatorname{deg} a, \operatorname{deg} b, \operatorname{deg} c\}<n_{0}(a b c)$. Here $n_{0}(f):=\operatorname{deg}(\operatorname{rad} f)$, where $\operatorname{rad} f$ is the product of all distinct prime factors of the polynomial $f=f(x)$, i.e., $n_{0}(f)$ is the number of all distinct roots of $f$, and $n_{0}(0):=-\infty$. The "moral" of Mason's lemma is that the number of distinct prime divisors of the product of three coprime members is not very small if one of the factors is the sum of the other two. (The corresponding "abc conjecture" in number theory seems to be much more subtle.)

A quick proof of Mason's lemma is to observe that $f / \operatorname{rad} f$ divides the derivative $f^{\prime}$ (just write $f$ as the product of powers of prime factors). From $a+b=c$ and $a^{\prime}+b^{\prime}=c^{\prime}$, it is easy to check that $a b^{\prime}-a^{\prime} b=b^{\prime} c-b c^{\prime}$. Next, $c / \operatorname{rad} c$ divides both $c$ and $c^{\prime}$, hence $b^{\prime} c-b c^{\prime}$, and similarly $b / \operatorname{rad} b$. Also, $a / \operatorname{rad} a$ divides $a b^{\prime}-a^{\prime} b=b^{\prime} c-b c^{\prime}$. Since $a, b, c$ are coprime, so are $a / \operatorname{rad} a, b / \operatorname{rad} b, c / \operatorname{rad} c$. Therefore, their product divides $b^{\prime} c-b c^{\prime}$. Assume conversely that $\operatorname{deg} a \geq n_{0}(a b c)$. This implies

$\operatorname{deg}(a b c / \operatorname{rad}(a b c))=\operatorname{deg}(a b c)-n_{0}(a b c) \geq \operatorname{deg}(a b c)-\operatorname{deg} a=\operatorname{deg}(b c)>\operatorname{deg}\left(b^{\prime} c-b c^{\prime}\right)$.

But only the zero polynomial (of degree $-\infty$ ) can be divisible with a polynomial of higher degree. So, $b^{\prime} c-b c^{\prime}=a b^{\prime}-a^{\prime} b=0$. Since $a$ divides $a b^{\prime}$, it follows that $a$ divides $b a^{\prime}$, but since $\operatorname{gcd}(a, b)=1$, it follows that $a$ divides $a^{\prime}$, and this in turn implies $a^{\prime}=0$, and similarly $b^{\prime}=c^{\prime}=0$, i.e., all $a, b$ and $c$ are constants. This contradiction proves the lemma.

Now the polynomial "FLT" follows easily, since $f^{n}+g^{n}=h^{n}$ implies by Mason's lemma that the degrees of $f^{n}, g^{n}, h^{n}$ are less than or equal to $\operatorname{deg} f+\operatorname{deg} g+\operatorname{deg} h-1$ (by assumption this is $\geq 0$ ). Addition of three inequalities yields

$$
n(\operatorname{deg} f+\operatorname{deg} g+\operatorname{deg} h) \leq 3(\operatorname{deg} f+\operatorname{deg} g+\operatorname{deg} h)-3,
$$

implying $n \leq 2$. More generally, by using Mason's lemma, it is easy to show that the polynomial Diophantine equation $f p+g q=h r$, where $2 \leq p \leq q \leq r$, can have solutions only if $(p, q, r)=(2,2, r),(2,3,3),(2,3,4)$ or $(2,3,5)$.

\subsection{Higher dimensional Candido's identity and concluding remarks}

Now, in 3D and higher dimensions, a similar "zero-volume" method could be applied as well. Heron's formula for the volume $V$ of an $n$-simplex $A_{0} A_{1} \ldots A_{n}$ in terms of its 
edge lengths $d_{i, j}=d\left(A_{i}, A_{j}\right)$ is also known as the Cayley-Menger formula from 1928:

$$
(-1)^{n+1} 2^{n}(n ! V)^{2}=\operatorname{det}\left(C M_{n}\right),
$$

where $C M_{n}$ is the $(n+2) \times(n+2)$ matrix obtained from the $(n+1) \times(n+1)$ matrix $M=\left(d_{i, j}^{2}\right)$ by bordering $M$ with a top row $(0,1, \ldots, 1)$ and a left column $(0,1, \ldots, 1)^{T}$; references for proof are in [4], see also [5, 6].

So, the volume $\operatorname{vol}(T)$ of a tetrahedron $T$ whose basis is a triangle with edge lengths $U, V$ and $W$ and opposite (disjoint) to them $u, v$ and $w$, respectively, is given by (Piero della Francesca, 15th century):

$$
\begin{aligned}
(12 \operatorname{vol}(T))^{2}= & \left(U^{2}+u^{2}\right)\left(-U^{2} u^{2}+V^{2} v^{2}+W^{2} w^{2}\right)+\left(V^{2}+v^{2}\right)\left(U^{2} u^{2}-V^{2} v^{2}+W^{2} w^{2}\right) \\
& +\left(W^{2}+w^{2}\right)\left(U^{2} u^{2}+V^{2} v^{2}-W^{2} w^{2}\right)-(U v w)^{2}-(u V w)^{2}-(u v W)^{2} \\
& -(U V W)^{2},
\end{aligned}
$$

or as Euler presented it in 1752:

$$
\begin{aligned}
(12 \operatorname{vol}(T))^{2}= & (2 u v w)^{2}-u^{2}\left(v^{2}+w^{2}-U^{2}\right)^{2}-v^{2}\left(w^{2}+u^{2}-V^{2}\right)^{2}-w^{2}\left(u^{2}+v^{2}-W^{2}\right)^{2} \\
& +\left(v^{2}+w^{2}-U^{2}\right)\left(w^{2}+u^{2}-V^{2}\right)\left(u^{2}+v^{2}-W^{2}\right) .
\end{aligned}
$$

The polynomials on the right hand sides do not factorize (see [4]), but the (1,1)-minor of $\operatorname{det}(C M 3)$, which is the squared area of the triangle with side lengths $U u, V v$ and $W w$ factorizes. In fact, we have the following product formula for volume (see, e.g., [5] or $[6])$ :

$(24 R \operatorname{vol}(T))^{2}=(U u+V v+W w)(-U u+V v+W w)(U u-V v+W w)(U u+V v-W w)$,

where $R$ is the circumradius of the tetrahedron $T$.

If one of the last three factors in the above formula is zero, then $T$ is a circumscribed quadrilateral by Ptolemy's theorem.

There are many interesting consequences of the fact that $\operatorname{vol}(T)=0$. For example, if we take any point in the plane of an equilateral triangle with side length $a$ and distances $d_{0}, d_{1}$ and $d_{2}$ to the vertices of the triangle, then the volume of this planar four-point configuration is zero, and the following quartic Candido type identity holds (e.g., from Euler's volume formula):

$$
\left(a^{2}+d_{0}^{2}+d_{1}^{2}+d_{2}^{2}\right)^{2}=3\left(a^{4}+d_{0}^{4}+d_{1}^{4}+d_{2}^{4}\right) .
$$


A simple (integer) example $\left(a, d_{0}, d_{1}, d_{2}\right)$ is $(8,3,5,7)$, and another one is $(112,57,65,73)$. If a point is taken in space, but out of the plane of the triangle, then the inequality $>$ occurs instead of equality $=$.

Similarly, if we take a point in a regular tetrahedron of edge length $a$, with distances $d_{0}, d_{1}, d_{2}$ and $d_{3}$ to the vertices of the tetrahedron, then we have the following Candido type identity (think of the methane molecule $\mathrm{CH}_{4}$, where the atom $\mathrm{C}$ is in the center or perhaps displaced a bit from the center of the $H$-tetrahedron):

$$
\left(a^{2}+d_{0}^{2}+d_{1}^{2}+d_{2}^{2}+d_{3}^{2}\right)^{2}=4\left(a^{4}+d_{0}^{4}+d_{1}^{4}+d_{2}^{4}+d_{3}^{4}\right) .
$$

This is useful in chemistry, and can serve as an equation for unknown $a$ or one of $d_{i}$ 's.

In general, for a regular $n$-simplex with edge length $a$ and a point in the hyperplane spanned by it, with distances to the vertices $d_{i}$ for $i=0,1, \ldots, n$, we have (from the Cayley-Menger formula):

$$
\left(a^{2}+\sum d_{i}^{2}\right)^{2}=(n+1)\left(a^{4}+\sum d_{i}^{4}\right) .
$$

Note that the ordinary Candido identity is the 1-dimensional case of the this formula.

The above identity is reminiscent of Soddy's mutually kissing (tangent) spheres formula: if $n+2$ spheres of dimension $n-1$ in the Euclidean $n$-space kiss each other, then $\left(\sum c_{i}\right)^{2}=n \sum c_{i}^{2}$, where $c_{i}=1 / r_{i}$ is the curvature of the $i$-th sphere, $i=1,2, \ldots, n+2$. In particular, for $n=2$, this is the famous Descartes' four circle theorem from 1643. No wonder, it is also a consequence of Heron's formula.

The "zero-volume" geometric procedure can, in principle, be applied to any $n$ simplex. However, the Cayley-Menger polynomial is irreducible in dimension $n \geq 3$ (see [4]); in other words, there is no Heron's type product formula for volume of a simplex in terms of edges in higher dimensions, and hence no general symmetric identities of Candido's type, except in special cases as above. For a general $n$-simplex there are some sharp symmetric inequalities for volume $V$ in terms of edges $d_{i, j}$. For example (see $[7])$,

$$
2^{n}(n ! V)^{2} \leq(n+1)\left(\prod d_{i, j}\right)^{4 /(n+1)},
$$

with equality if and only if the simplex is regular.

In dimension 3 for a tetrahedron $T$ there is also Kahan's product formula for volume from 1985 (available online). As before, let $u, U ; v, V$ and $w, W$, respectively, be pairs 
of opposite side lengths of $T$, where $u, v$ and $w$ have a common vertex. Then the volume $\operatorname{vol}(T)$ of $T$ is given by:

$$
\left(3 \cdot 2^{6} \cdot u v w \cdot \operatorname{vol}(T)\right)^{2}=(-a+b+c+d)(a-b+c+d)(a+b-c+d)(a+b+c-d),
$$

where $a=\sqrt{x Y Z}, b=\sqrt{X y Z}, c=\sqrt{X Y z}, d=\sqrt{x y z}$, and $X=(-U+v+w)(U+v+w)$, $x=(U-v+w)(U+v-w)$ and similarly $Y=(u-V+w)(u+V+w), y=(-u+V+$ $w)(u+V-w), Z=(u+v-W)(u+v+W), z=(-u+v+W)(u-v+W)$.

For instance, if $T$ is a regular tetrahedron with side length $t$, then $a=b=c=3 t^{3}$, and $d=t^{3}$; next if $T$ is a corner of a brick with edges $u, v, w$, then $X=x=2 v w$, $Y=y=2 u w, Z=z=2 u v$, and $a=b=c=d=2 u v w \sqrt{2}$, while if $u=v=w=t \sqrt{3}$, and $U=V=W=3 t$, then $a=b=c=9 t^{3}$ and $d=27 t^{3}$, and $T$ is an equilateral triangle and its center $(\mathrm{so}, \operatorname{vol}(T)=0)$. Let us mention here that it is an open problem ("perfect Euler's brick problem") whether there exist integers $u, v, w$ as brick edge lengths such that plane diagonals $U, V, W$ and space diagonal $\sqrt{u^{2}+v^{2}+w^{2}}$ all have integer lengths.

A Heronian tetrahedron example is $U, V, W=25,39,56$ and $u, v, w=120,160,153$, having face areas area $(25,39,56)=420$, area $(25,153,160)=1404$, area $(39,120,153)=$ 1872 , area $(56,120,160)=2688$, and volume 8064. $X=2^{6} 3^{4} 13^{2}, x=2^{6} 3^{2}, Y=2^{3} 3^{3} 13^{2}$, $y=2^{4} 3^{3}, Z=2^{8} 3 \cdot 7^{2}, z=2^{9} \cdot 3$, then $a=2^{8} 3^{3} 7 \cdot 13 \sqrt{2}, b=2^{9} 3^{4} 7 \cdot 13^{2}, c=2^{9} 3^{4} 13^{2}$, $\mathrm{d}=2^{9} 3^{3} \sqrt{2}$. (U,V,W and $u, v, w$ are length edges of a tetrahedron iff they obey all triangle inequalities and the (1,1)-minor of $C M_{3}$ has positive determinant, for a nice proof see [8].)

From six facial difference products (of three faces, not of the base $U V W$-triangle), as positive independent variables, we can reconstruct all six edge lengths, e.g.,

$$
\begin{gathered}
(2 u)^{2}=(Y+y)(Z+z) /(X+x), \\
(2 U)^{2}=\left(X(Y+y-Z-z)^{2}+x(Y+y+Z+z)^{2}\right) /(Y+y)(Z+z),
\end{gathered}
$$

etc.

Suppose, for instance, that in Kahan's formula $b+c+d=a$, i.e. $\sqrt{X y Z}+\sqrt{X Y z}+$ $\sqrt{x y z}=\sqrt{x Y Z}$. By substituting $u, v, w, U, V, W$ we obtain the sum of three square roots, equals also to a square root and each square root has 6 factors. The volume of $T$ is then zero, and we have as a result of "freeing the square roots" the following polynomial identity of degree 6 (from Euler's volume expression):

$$
\begin{aligned}
(2 u v w)^{2} & +\left(v^{2}+w^{2}-U^{2}\right)\left(w^{2}+u^{2}-V^{2}\right)\left(u^{2}+v^{2}-W^{2}\right) \\
& =u^{2}\left(v^{2}+w^{2}-U^{2}\right)^{2}+v^{2}\left(w^{2}+u^{2}-V^{2}\right)^{2}+w^{2}\left(u^{2}+v^{2}-W^{2}\right)^{2} .
\end{aligned}
$$


From the product formula it is also equivalent to

$$
(-u U+v V+w W)(u U-v V+w W)(u U+v V-w W)=0,
$$

and this is again cubic Candido's identity for quantities $u U, v V$ and $w W$.

An interesting special case is when opposite edges are equal. Then $T$ is called equifacetal (or disphenoid). In this case we get the same identity as for the Pythagorean theorem, $e_{1}^{3}+8 e_{3}=4 e_{1} e_{2}$ in variables $A 2, B 2, C 2$, where $A=u=U$ etc.

Back to Kahan's formula, $\operatorname{vol}(T)=0$ (assuming $u v w \neq 0$ ) if and only if

$$
D 4(a, b, c, d):=(-a+b+c+d)(a-b+c+d)(a+b-c+d)(a+b+c-d)=0 .
$$

In terms of elementary symmetric functions $e_{1}=e_{1}(a, b, c, d)$, and similarly $e_{2}, e_{3}, e_{4}$, we have that $\operatorname{vol}(T)=0$, if and only if $e_{1}\left(e_{1}^{3}+8 e_{3}-4 e_{1} e_{2}\right)=16 e_{4}$.

As a combinatorial example, consider Tribonacci numbers $T_{n}$, i.e. $0,0,1,1,2,4,7,13,24,44, \ldots$ given by $T_{0}=T_{1}=0, T_{2}=1, T_{n}+2=T_{n}+1+T_{n}+T_{n-1}$. $T_{n+2}$ is the number of compositions of $n$ as sums of 1 's, 2 's and 3's. Note that $T_{n+1} / T_{n}$ ,as $n \rightarrow \infty$, tends to the "metallic" number $t \approx 1.83929 \ldots$ which satisfies $t^{3}=t^{2}+t+1$. If $e_{i}$ are symmetric functions of $T_{j}$ 's then we get again "for free" the identity for Tribonacci numbers of Candido's type in the above form.

Finally, we describe one more volume formula where the ordinary Candido form occurs. Let $T=A_{0} A_{1} A_{2} A_{3}$ be a tetrahedron, let $F_{i}$ be the face against $A_{i}, i=0,1,2,3$, and let $(i k)$ be the face angle of $T$ at the vertex $A_{k}$ in $F_{i},(i \neq k)$. Let $R_{i}$ be the circumradius of $F_{i}$ and $R$ the circumradius of $T$. Then (see [9]) the volume of $T$ is given by $3 R \cdot \operatorname{vol}(T)=R_{1}^{2} R_{3}^{2} \sqrt{N}$, where $N=2\left[(A B)^{2}+(B C)^{2}+(C A)^{2}\right]-\left(A^{4}+B^{4}+C^{4}\right)$, and $A=\sin (32) \sin (10), B=\sin (31) \sin (20) \sin (12) / \sin (21), C=\sin (30) \sin (12)$. Recall that the ordinary Candido identity is equivalent to $N:=2\left[(A B)^{2}+(B C)^{2}+(C A)^{2}\right]-$ $\left(A^{4}+B^{4}+C^{4}\right)=0$. We see that the Candido's form on the right hand side occurs in this volume formula. Clearly, $R \cdot \operatorname{vol}(T)=0$ if and only if $N$ is 0 , because in this case $R$ becomes infinite (and $R_{1}$ and $R_{3}$ can be considered constant). The vanishing of Candido' $\mathrm{s}$ form $N$ (this time in trigonometric terms of face angles) shows that $\mathrm{T}$ is degenerate. A similar formula ([9]), but a bit more involved, can also be given in terms of inradii of faces, circumradius $R$ and face angles, again in a Candido-type form.

To conclude, we see that a simple addition of two elements is via Candido's identity transformed into a symmetric equation involving sums of squares, cubes, fourth powers or other symmetric functions of summands and the sum. It is amazing that a purely algebraic formula has a simple geometric proof, and how it applies to various situations in combinatorics, algebra, geometry and analysis. 


\section{References}

[1] C. Alsina and R.B. Nelsen, Candido's identity, Math. Magazine 80 (2007) 226-228.

[2] T. Koshy, Candido's Identity and the Pell Family, in Pell and Pell-Lucas Numbers with Applications, Springer Verlag, New York, 2014.

[3] S. Kurz, On the generation of Heronian triangles, arXiv:1401.6150v.1.

[4] C. D'Andrea and M. Sombra, The Cayley-Menger determinant is irreducible for $n \geq 3$, Siberian Math. J. 46 (2005) 71-76.

[5] M. Fiedler, Matrices and Graphs in Geometry, Cambridge University Press, Cambridge, 2011.

[6] M. Berger, Geometry, Springer Verlag, Berlin, 1987.

[7] D. Veljan, The sine theorem and inequalities for volumes of simplices and determinants, Lin. Alg. Appl. 219 (1995) 79-91.

[8] K. Wirth and A.S. Dreiding, Edge lengths determining tetrahedrons, Elem. Math. 64 (2009) 160-170.

[9] A. Bhattacharyya and B. Pal, Volume of tetrahedron revisited, Demonstratio Math. 47 (2014) 253-257. 
